Canad. Math. Bull. Vol. 19 (1), 1976

\title{
CENTRALIZING AUTOMORPHISMS OF PRIME RINGS
}

\author{
BY \\ JOSEPH H. MAYNE ${ }^{1}$
}

\begin{abstract}
Let $R$ be a prime ring and $T$ be a nontrivial automorphism of $R$. If $x x^{T}-x^{T} x$ is in the center of the ring for every $x$ in $R$, then $R$ is a commutative integral domain.
\end{abstract}

An additive mapping $L$ of a ring $R$ to itself is called centralizing if $x(x L)-(x L) x$ is in the center of $R$ for every $x$ in $R$. In [4] Posner showed that a prime ring must be commutative if it has a nontrivial centralizing derivation (see [1] for another proof). In this note the analogous result for a centralizing automorphism is proved.

THEOREM. If $R$ is a prime ring with a nontrivial centralizing automorphism, then $R$ is a commutative integral domain.

This generalizes the results of Divinsky [2] and Luh [3]. Divinsky showed that a simple ring is commutative if it has a nontrivial automorphism $T$ such that $x x^{T}=$ $x^{T} x$ for all $x$ in the ring and Luh extended this result to prime rings.

Let $[x, y]=x y-y x$ and note that $[x, y z]=y[x, z]+[x, y] z$. Assume that $R$ is a prime ring and let $Z$ be the center of $R$. The next two lemmas will be used in the proof of the theorem.

LEMMA 1. [3] Let T be a nontrivial automorphism of $R$. If $\left[x, x^{T}\right]=0$ for all $x$ in $R$, then $R$ is commutative.

Proof. Linearizing $\left[x, x^{T}\right]=0$ gives $\left[x, y^{T}\right]=\left[x^{T}, y\right]$ and thus $\left[x,(x y)^{T}\right]=$ $\left[x^{T}, x y\right]$. But $\left[x,(x y)^{T}\right]=x^{T}\left[x, y^{T}\right]$ and $\left[x^{T}, x y\right]=x\left[x^{T}, y\right]=x\left[x, y^{T}\right]$. Thus $\left(x-x^{T}\right)\left[x, y^{T}\right]=0$ and since $T$ is an automorphism $\left(x-x^{T}\right)[x, z]=0$ for all $x$ and $z$ in $R$. Since $y[x, z]=[x, y z]-[x, y] z,\left(x-x^{T}\right) R[x, z]=0$. If $x \neq x^{T}$, then $x$ is in the center since $R$ is prime. Since $T$ is nontrivial, there must be at least one $x$ such that $x \neq x^{T}$. Suppose $y$ is not in the center of $R$. Then $x+y$ is not in the center and $y^{T}=y$, $(x+y)^{T}=x+y$. But then $x=x^{T}$ which is a contradiction. Hence $R$ is commutative.

LEMMA 2. If $x y=0$ and $x$ is a nonzero element in $Z$, then $y=0$.

Proof. If $x y=0$, then $z x y=x z y=0$ for all $z$ in $R$. Since $R$ is prime, and $x \neq 0$, $y$ must be 0 .

Proof of the theorem. Let $T$ be a nontrivial automorphism of $R$ such that $\left[x, x^{T}\right]$ is in $Z$ for all $x$ in $R$. The proof will consist of showing that $\left[x, x^{T}\right]=0$ for

(') A portion of the research for this paper was done while the author was a Faculty Research Participant at the Argonne National Laboratory. 
all $x$ in $R$ and then using Lemma 1 to conclude that $R$ is commutative. Linearization of $\left[x, x^{T}\right]$ in $Z$ gives

$$
\left[x, y^{T}\right]+\left[y, x^{T}\right] \text { is in } Z \text { for all } x \text { and } y \text { in } R,
$$

and thus

$$
\left[x,\left[x, y^{T}\right]+\left[y, x^{T}\right]\right]=0 \text { for all } x \text { and } y \text { in } R .
$$

Now $R$ is a prime ring so $R$ is either of characteristic two or $2 x=0$ implies $x=0$ for $x$ in $R$.

Suppose $R$ is not of characteristic two and let $y=x^{2}$ in (2). Then $0=\left[x, x,\left(x^{2}\right)^{T}\right]$ $\left.+\left[x^{2}, x^{T}\right]\right]=\left[x, 2 x^{T}\left[x, x^{T}\right]\right]+\left[x, 2 x\left[x, x^{T}\right]\right]=2\left[x, x^{T}\right]^{2}$. Hence $\left[x, x^{T}\right]^{2}=0$. By Lemma $2\left[x, x^{T}\right]=0$ for all $x$ in $R$ and thus $R$ is commutative.

Now suppose that $R$ is of characteristic two. Then $\left[x^{2}, x^{T}\right]=2 x\left[x, x^{T}\right]=0$ and $\left[\left(x^{T}\right)^{2}, x\right]=2 x^{T}\left[x, x^{T}\right]=0$. Let $y=x^{T}$ in (1), then $\left[x, x^{T T}\right]+\left[x^{T}, x^{T}\right]=$ $\left[x, x^{T T}\right]$ is in $Z$. Using the Jacobi identity (2) can be rewritten as

$$
\left[x,\left[y^{T}, x\right]\right]+\left[x^{T},[x, y]\right]=0 .
$$

Letting $y=x^{3} x^{T}$ in (3) gives

$$
\left[x,\left[\left(x^{3} x^{T}\right)^{T}, x\right]\right]+\left[x^{T},\left[x, x^{3} x^{T}\right]\right]=0 .
$$

Now $\left[x,\left[\left(x^{3} x^{T}\right)^{T}, x\right]\right]=\left[x,\left(x^{3} x^{T}\right)^{T} x+x\left(x^{3} x^{T}\right)^{T}\right]=\left[x^{2},\left(x^{3} x^{T}\right)^{T}\right]$. But expanding the last commutator gives

since

$$
\begin{aligned}
x\left[x,\left(x^{3} x^{T}\right)^{T}\right]+\left[x,\left(x^{3} x^{T}\right)^{T}\right] x \\
\quad=x\left(x^{T}\right)^{3}\left[x, x^{T T}\right]+x\left[x,\left(x^{T}\right)^{3}\right] x^{T T}+\left(x^{T}\right)^{3}\left[x, x^{T T}\right] x+\left[x,\left(x^{T}\right)^{3}\right] x^{T T} x \\
\quad=\left[x,\left(x^{T}\right)^{3}\right]\left[x, x^{T T}\right]+x\left(x^{T}\right)^{2}\left[x, x^{T}\right] x^{T T}+\left(x^{T}\right)^{2}\left[x, x^{T}\right] x^{T T} x
\end{aligned}
$$

Hence

$$
\left[x,\left(x^{T}\right)^{2}\right]=0 .
$$

Thus (4) reduces to

$$
\begin{aligned}
{\left[x,\left[\left(x^{3} x^{T}\right)^{T}, x\right]\right] } & =\left[x,\left(x^{T}\right)^{3}\right]\left[x, x^{T T}\right]+\left(x^{T}\right)^{2}\left[x, x^{T}\right]\left[x, x^{T T}\right] \\
& =2\left[x,\left(x^{T}\right)^{3}\right]\left[x, x^{T T}\right]=0 .
\end{aligned}
$$

$$
\left[x^{T},\left[x, x^{3} x^{T}\right]\right]=0 .
$$

But then $0=\left[x^{T}, x^{3}\left[x, x^{T}\right]\right]=\left[x^{T}, x^{3}\right]\left[x, x^{T}\right]$ and using $\left[x^{T}, x^{2}\right]=0$ results in

$$
x^{2}\left[x, x^{T}\right]^{2}=0 \text { for all } x \text { in } R .
$$

By Lemma 2 , if $\left[x, x^{T}\right] \neq 0$, then $x^{2}=0$. So assume $x^{2}=0$, then $\left(x^{T}\right)^{2}=0$ and $\left(x^{T T}\right)^{2}=0$. Now $\left(x^{T} x\right)\left(x x^{T}\right)=0$ and $\left[x, x^{T}\right]=x x^{T}+x^{T} x=z$ for some $z$ in $Z$. Therefore $\left(x x^{T}+z\right)\left(x x^{T}\right)=0$ and thus $\left(x x^{T}\right)^{2}=z\left(x x^{T}\right)$. If $\left(x x^{T}\right)^{2}=0$, then $z\left(x x^{T}\right)=$ 0 and so either $z=0=\left[x, x^{T}\right]$ or $x x^{T}=0$. But if $x x^{T}=0$, then $\left[x, x^{T}\right] x=\left(x^{T} x\right) x=0$ and hence $\left[x, x^{T}\right]=0$ or $x=0$. So from now on, assume that $x^{2}=0$ and $\left(x x^{T}\right)^{2} \neq 0$. 
Now (6) with $x x^{T}$ replacing $x$ implies that $\left[x x^{T},\left(x x^{T}\right)^{T}\right]=0$. Expanding gives $x\left[x^{T}, x^{T} x^{T T}\right]+\left[x, x^{T} x^{T T}\right] x^{T}=0$. If this equation is left multiplied by $x$, then $x\left[x, x^{T} x^{T T}\right] x^{T}=0$ and so $x x^{T}\left[x, x^{T T}\right] x^{T}+x\left[x, x^{T}\right] x^{T T} x^{T}=0$. But $x x^{T}\left[x, x^{T T}\right] x^{T}$ $=x\left(x^{T}\right)^{2}\left[x, x^{T T}\right]=0$. Thus $x\left[x, x^{T}\right] x^{T T} x^{T}=\left[x, x^{T}\right] x x^{T T} x^{T}=0$. If $\left[x, x^{T}\right] \neq 0$, then $x x^{T T} x^{T}=0$.

Thus $\left[x, x^{T T}\right] x^{T}=x^{T T} x x^{T}$, and so $x^{T T}\left[x, x^{T T}\right] x^{T}=\left(x^{T T}\right)^{2} x x^{T}=0$. Hence if $\left[x, x^{T T}\right] \neq 0$, then $x^{T T} x^{T}=0$. But this forces $x^{T} x=0$ and so $x=0$ or $\left[x, x^{T}\right]=0$. Suppose then that $\left[x, x^{T T}\right]=0$. Letting $y=x x^{T}$ in (2) results in $\left[x,\left[x^{T}, x x^{T}\right]+\right.$ $\left.\left[x,\left(x x^{T}\right)^{T}\right]\right]=0$. Thus $\left[x, x^{T}\left[x, x^{T}\right]+x^{T}\left[x, x^{T T}\right]+\left[x, x^{T}\right] x^{T T}\right]=0$. But then $\left[x, x^{T}\right]^{2}+2\left[x, x^{T}\right]\left[x, x^{T T}\right]=\left[x, x^{T}\right]^{2}=0$. Therefore $\left[x, x^{T}\right]=0$ for all $x$ in $R$ and by Lemma $1, R$ is commutative.

\section{REFERENCES}

1. R. Awtar, "On a Theorem of Posner," Proc. Cambridge Philos. Soc. 73 (1973), 25-27.

2. N. Divinsky, "On commuting automorphisms of rings," Trans. Roy. Soc. Canada, Sect. III, 49 (1955), 19-22.

3. J. Luh, "A note on commuting automorphisms of rings," Amer. Math. Monthly 77 (1970), 61-62.

4. E. Posner, “Derivations in prime rings," Proc. Amer. Math. Soc. 8 (1957), 1093-1100.

\section{Applied Mathematics Division}

ArgonNe National Laboratory

ARGONNE, ILLINOIS 60439

U.S.A.
Department of Mathematics LOYOLA UNIVERSITY Chicago, Illinois 60626 U.S.A. 